Proyecciones

Vol. 25, $\mathrm{N}^{o}$ 3, pp. 231-236, December 2006.

Universidad Católica del Norte

Antofagasta - Chile

\title{
UNE REMARQUE SUR LA TRACE DE LA TORSION ET LE TENSEUR DE RICCI
}

\author{
F. LESCURE \\ Universitée de Lille 1, France
}

Received : July 2006. Accepted : September 2006

\begin{abstract}
Résumé: On donne une formule qui raccorde la trace de la torsion, le tenseur de Ricci et l'application exponentielle d'une connexion pour laquelle une forme volume est à dérivée covariante nulle. Ce résultat élémentaire répond à une question souvent posée.
\end{abstract}


Soit $M$ une variété différentiable munie d'une connexion linéaire et donc d'un opérateur de dérivation covariante qui, à tout champ de vecteurs $X$, fait correspondre une 1-forme $\nabla X$ à valeurs dans le fibré tangent. $\mathrm{Au}$ moyen d'une carte locale cette connexion est déterminée par ses symboles de Christoffel de première espèce $\Gamma_{i}{ }^{k}{ }_{j}$ tels que, en faisant la convention d'Einstein, les composantes de $\nabla X$ soient données par la formule: $\nabla_{i} X^{k}=$ $\partial_{i} X^{k}+\Gamma_{i}{ }_{j}{ }_{j} X^{j}$. Dans tout ce qui suivra nous ferons d'ailleurs souvent la convention d'Einstein sans même le préciser expréssément.

La quantité: $T(X, Y)=\nabla_{X} Y-\nabla_{Y} X-[X, Y]$ est "tensorielle" et $T$ est la 2-forme de torsion à valeurs dans le fibré tangent. Dans un système de coordonnées locales on écrit classiquement ses composantes: $T_{i}{ }_{j}{ }_{j}=$ $\Gamma_{i}{ }^{k}{ }_{j}-\Gamma_{j}{ }_{j}{ }_{i}$. Modifiant un peu le vocabulaire de [1], on appellera 1-forme de torsion la 1-forme $\mathbf{T}$ à valeurs dans le fibré vectoriel End $(T M)$ qui, à un champ de vecteurs $X$, associe le champ d'endomorphismes $\mathbf{T}(X)$ qui à un champ $Y$ fait correspondre $\mathbf{T}(X)(Y)$ def $T(X, Y)$. Et, par contre nous appellerons trace de la 1-forme de torsion la forme $\theta$ définie par: $\theta(\mathrm{X})=$ $\operatorname{tr}((\mathbf{T})(\mathbf{X}))$. C'est une 1-forme de composantes covariantes: $(\theta)_{\mathbf{i}}=\mathbf{T}_{\mathbf{i}} \mathbf{k}_{\mathbf{k}}$. Cette trace apparait implicitement dans les travaux de Mrs Cherrier et Hanani; le résultat le plus marquant à son sujet est évidemment, dans le cas de la connexion de Chern, l'existence ([1]) de métriques standards dans chaque classe conforme. $c f$ aussi $[2]^{1}$ et [4] pour des développements à son sujet.

Enfin la courbure: $R(X, Y) Z$ def $\nabla_{X} \nabla_{Y} Z-\nabla_{Y} \nabla_{X} Z-\nabla_{[X, Y]} Z$, est ici comprise comme une 2 -forme à valeurs dans End $(T M)$, et $R_{j, k_{0} \ell_{0}}$ sont les composantes du tenseur 1-fois covariant et 1-fois contravariant $R\left(\frac{\partial}{\partial x^{k_{0}}}, \frac{\partial}{\partial x^{l_{0}}}\right)$ Si $X$ et $Y$ sont deux champs de vecteurs, ils définissent une section dans End $(T M)$ à savoir: $Z \mapsto R(Z, Y) X$. La trace de cet endomorphisme est une quantité $\mathbf{r}(X, Y)$ qui dépend bilinéairement de ses arguments, et $\mathbf{r}$, interprété comme tenseur deux fois covariant s'appelle le tenseur de Ricci de la connexion de composantes: $\mathbf{r}_{i j}=R_{i, k j}^{k}$. Dans le cas particulier d'une connexion de Levi-Civita ce tenseur de Ricci est symétrique mais tel nàst point le cas en général. L'objet de la présente remarque est de répondre à une question souvent posée en écrivant une formule où interviennent toutes ces quantités et qui en donne une sig-

La moitié de l'opérateur de Dirac différe de $\bar{\partial}+\bar{\partial}^{*}$ d'une quantité où intervient clairement cette trace 
nification géométrique. Dans le cas plus particulier de la connexion de Levi-Civita on retrouve une interprétation géométrique du tenseur de Ricci, inévitablement connue, mais dont, curieusement, léxplicitation semble faire défaut dans la littérature.

Sans supposer, pour commencer, l'existence d' une forme volume vol $_{M}$ à $\nabla v o l_{M}=0$. ce qui exige une condition d'intégrabilité ${ }^{2}$ nous allons, au moyen des coordonnées géodésiques, montrer quelques identités utiles.

Considérons un point $m_{0} \in M$. Si on choisit un système de coordonnées locales centrée en $m_{0}$, et donc des symboles de Christoffel pour représenter la connexion, un chemin paramétré de classe $C^{2}$ qui passe par $m_{0}$ "au temp $t=0$ " est donné par $t \mapsto \mathbf{x}(t)$ def $\left(x^{1}(t), \ldots, x^{m}(t)\right)$, avec $x^{k}(0)=0$. La géodésique qui passe par $m_{0}$ en $t=0$ vérifie l'equation différentielle $\ddot{x}^{k}(t)+\Gamma_{i}{ }_{j}{ }_{j} \dot{x}^{i}(t) \dot{x}^{j}(t)=0$ (où, dans cette équation il s'agit des valeurs prises par le symbole de Christoffel au point de coordonnées locales $\mathbf{x}(t)$ ), et $\mathbf{x}(0)=0$. Si $\mathbf{u} \in T_{0} M$ est assez petit la solution de l'équation qui précède avec $\dot{x}(0)=\mathbf{u}$ et $x(0)=0$ a un domaine d'existence assez grand pour que l'on puisse considérer les coordonnées locales $\mathbf{x}(1)$ d'un point $\exp (\mathbf{u}) \in \mathbf{M}$ dans le domaine de la carte. On sait qu'alors exp envoie difféomorphiquement un voisinage de $0 \in T_{m 0} M$ sur un voisinage de $m_{0} \in$ $M$.

La "carte géodésique centrée en $m_{0}$ " est par définition la transformation $\exp ^{-} 1$. Choisissons un repère dans l'espace tangent; la carte géodésique nous donne un véritable système de coordonnées locales. Un point $\mathbf{x} \in$ $T_{m_{0}} M$ sera identifié au $m$-uplet de ses coordonnées $\mathbf{x}=\left(x^{1}, \ldots, x^{m}\right)$. Les symboles de Christoffel deviennent des fonctions de $x$, et le fait que les géodésiques qui passent par $m_{0}$ s'interprètent comme les "rayons" $t \mapsto t \mathbf{u}$ montre qu'en $x=t \mathbf{u}$ on a:

$$
\Gamma_{i}{ }_{j}{ }_{j}(\mathbf{x}) x^{i} x^{j}=t^{2} \Gamma_{i}{ }^{k}{ }_{j}(t \mathbf{u}) \mathbf{u}^{i} \mathbf{u}^{j}=0 .
$$

En résulte classiquement que $\Gamma_{i}{ }^{k}{ }_{j}(0)=\frac{1}{2} T_{i}{ }^{k}$, mais aussi, en observant que la quantité identiquement nulle écrite en (1) a pour terme d'ordre 3 en $t$ la quantité $t^{3} \partial_{\ell} \Gamma_{i}{ }^{k}{ }_{j}(0) \mathbf{u}^{\ell} \mathbf{u}^{i} \mathbf{u}^{j}$, que le symétrisé ${ }^{3}$ par rapport à ses trois

\footnotetext{
${ }^{2}$ qui, en coordonnées locales, s'exprime par la fermeture de la 1-forme non intrinséquement définie, $\Gamma_{i}{ }_{k}{ }_{k} d x^{i}$.

${ }^{3}$ Une forme trilinéaire $\Phi$ symétrique est complétement déterminée par la fonction polynomiale homogène: $\mathbf{u} \rightarrow \Phi(\mathbf{u}, \mathbf{u}, \mathbf{u})$
} 
indices covariants de la quantité $\partial_{\ell} \Gamma_{i}{ }^{k}{ }_{j}(0)$ est nul. Mais alors l'égalité:

$$
\left[\partial_{k} \Gamma_{j}^{\ell}{ }_{i}+\partial_{j} \Gamma_{i k}^{\ell}+\partial_{i} \Gamma_{k}^{\ell}{ }_{j}\right] \mathbf{u}^{i} \mathbf{u}^{j}=
$$

$$
\frac{1}{2}\left[\partial_{k} \Gamma_{j}{ }_{i}{ }_{i}+\partial_{j} \Gamma_{i}^{\ell}{ }_{k}{ }+\partial_{i} \Gamma_{k}{ }_{j}{ }_{j}+\partial_{k} \Gamma_{i}^{\ell}{ }_{j}+\partial_{i} \Gamma_{j}{ }_{k}{ }_{k}+\partial_{j} \Gamma_{k}{ }_{i}\right] \mathbf{u}^{i} \mathbf{u}^{j}
$$

montre qu'en $m_{0}$ le premier membre est nul. Par contraction des indices $\ell$ et $k$ on en déduit en l'origine l'égalité:

$$
\left[\partial_{\ell} \Gamma_{j}^{\ell}{ }_{i}+\partial_{j} \Gamma_{i}^{\ell}{ }_{\ell}+\partial_{i} \Gamma_{\ell}^{\ell}{ }_{j}\right] \mathbf{u}^{i} \mathbf{u}^{j}=0
$$

On va déduire de (3) une expression en l'origine du tenseur de Ricci qui nous sera utile. En effet dans l'égalité:

$$
\mathbf{r}(\mathbf{u}, \mathbf{u})=\left(\partial_{\ell} \Gamma_{j}{ }_{i}{ }_{i}-\partial_{j} \Gamma_{\ell}{ }_{\ell}{ }_{i}\right) \mathbf{u}^{i} \mathbf{u}^{j}+\left(\Gamma_{\ell}{ }_{k} \Gamma_{j}{ }_{i}{ }_{i}-\Gamma_{j}{ }_{k}{ }_{k} \Gamma_{\ell}{ }^{k}{ }_{i}\right) \mathbf{u}^{i} \mathbf{u}^{j},
$$

on voit qu'en l'origine le deuxième terme du deuxième membre se simplifie puisque le symbole de Christoffel y est antisymétrique en ses indices covariants et qu'aussi: $-\Gamma_{j}{ }_{k}{ }_{k} \Gamma_{\ell}{ }_{i}{ }_{\mathbf{u}^{i}} \mathbf{u}^{j}=\frac{1}{4}\left(\mathbf{u}^{i} T_{i}{ }^{k} \ell\right)\left(\mathbf{u}^{j} T_{j}{ }_{k}{ }\right)=\frac{1}{4} \operatorname{tr} \mathbf{T}(\mathbf{u})^{\mathbf{2}}$. Quand au premier membre, on voit par (3) qu'il vaut aussi en l'origine:

$$
\left[\partial_{\ell} \Gamma_{j}^{\ell}{ }_{i}-\partial_{j} \Gamma_{\ell}^{\ell}{ }_{i}\right] \mathbf{u}^{i} \mathbf{u}^{j}=\left[-\partial_{i} \Gamma_{j}^{\ell} \ell_{\ell}-2 \partial_{j} \Gamma_{\ell}^{\ell}{ }_{i}\right] \mathbf{u}^{i} \mathbf{u}^{j}
$$

Mais l'identité $\partial_{i}\left(\Gamma_{j}^{\ell} \ell-\Gamma_{\ell}^{\ell} j\right)-\Gamma_{i}{ }^{\nu}{ }_{j}(\theta)_{\nu}=\nabla_{i}(\theta)_{j}$, montre, qu'en l'origine de la carte géodésique, ce premier membre vaut aussi $\left(-3 \partial_{i} \Gamma_{j}^{\ell} \ell+2 \nabla_{i}(\theta)_{j}\right) \mathbf{u}^{i} \mathbf{u}^{j}$. Si bien que (4) et ce qui précède permettent d'écrire:

$$
\mathbf{r}(\mathbf{u}, \mathbf{u})=\left[-3 \partial_{i} \Gamma_{j}^{\ell} \ell+2 \nabla_{i}(\theta)_{j}\right] \mathbf{u}^{i} \mathbf{u}^{j}+\frac{1}{4} \operatorname{tr} \mathbf{T}(\mathbf{u})^{2}
$$

Soit maintenant une forme volume $\operatorname{vol}_{M}$ sur $M$. Dans la carte géodésique, elle s'interprète ( $\mathrm{cf}[3]$ ) comme un tenseur $m$-fois covariant complètement antisymétrique $v_{i_{1}, \ldots, i_{m}}$. On considère le scalaire $v$ def $v_{1, \ldots, m}$, de telle manière que $\operatorname{vol}_{M}=v d x^{1} \wedge \ldots \wedge d x^{m}=\frac{1}{m !} v_{i_{1}, \ldots, i_{m}} d x^{i_{1}} \wedge \ldots \wedge d x^{i_{m}}$. Dire que: $\nabla v^{\prime} l_{M}=0$ s'xprime par les $m$ égalités:

$$
\nabla_{j} v_{1, \ldots, m}=\partial_{j} v_{1, \ldots, m}-\sum_{\ell} \Gamma_{j}^{\nu} \ell v_{1, \ldots, \ell-1, \nu, \ell+1, \ldots, m}=\partial_{j} v-\Gamma_{j}^{\ell} \check{v}=0
$$


Avec, en l'origine, l'expression donnée plus haut des symboles de Christoffel, on voit donc qu'on y obtient déjé l'égalité:

$$
\partial_{j} v(0)=\frac{1}{2} \theta_{j}(0) v(0)
$$

De plus, en dérivant la derniére des égalités de (6), on voit que l'on obtient aussi l'égalité:

$$
\partial_{i j} v=\partial_{i} \Gamma_{j}^{\ell} \ell v+\Gamma_{j}^{\ell} \ell \partial_{i} v=\left(\partial_{i} \Gamma_{j}^{\ell} \ell+\Gamma_{i}^{\ell} \ell \Gamma_{j}^{\ell} \ell\right) v
$$

Mais, en reportant dans cette formule, d'une part l'expression de $\partial_{i} \Gamma_{j} \ell_{\ell} \mathbf{u}^{i} \mathbf{u}^{j}$ donnée par l'égalité (5), d'autre part la valeur du symbole de Christoffel en l'origine on y trouve le déve loppement de Taylor:

$$
\begin{gathered}
v(\mathbf{u})= \\
v(0)\left\{1+\frac{1}{2}(\theta)(\mathbf{u})+\frac{1}{8} \theta(\mathbf{u})^{2}+\frac{1}{3 !}\left[2 \nabla_{i} \theta_{j} \mathbf{u}^{i} \mathbf{u}^{j}+\frac{1}{4} \operatorname{tr} \mathbf{T}(\mathbf{u})^{2}-\mathbf{r}_{i j} \mathbf{u}^{i} \mathbf{u}^{j}\right]+o\left(|\mathbf{u}|^{2}\right)\right\}
\end{gathered}
$$

Indiquons aussi, qu'en sus sus de la connexion de Levi-Civita pour laquelle $\mathbf{T}=(\theta)=\mathbf{0}$ il existe, dans le cas d'une métrique hermitienne sur une variété complexe, une connexion dont la moyenne avec la connexion de Chern est la premiére connexion hermitienne au sens de [3] et pour laquelle [2] le tenseur $\mathbf{T}$ est à valeurs antisymétriques, ce qui implique que $\theta=0$. La formule se simplifie et en sus $\operatorname{tr} \mathbf{T}(\mathbf{u})^{2}$ est alors une forme quadratique négative.

\section{References}

[1] Gauduchon P. La 1-forme de torsion d'une variété hermitienne compacte. Math. Ann. 267, pp. 495-518, (1984).

[2] Gauduchon P. Hermitian connections and Dirac Operators Bolletino U. M. I. (7) 11-B, Suppl. fasc. 2, pp. 257-288, (1997). 
[3] Lichnerowicz A. Théorie globale des connexions et des groupes d'holonomie. Edizioni Cremonese, Roma (1962).

[4] Yachou A. La variation de la $L^{2 r}$-norme de la 1 -forme de torsion sur une variété hermitienne compacte. Indagationes Mathematicae. NorthHolland., (1998).

\section{Francois Lescure}

UFR de Mathématiques

Universitée de Lille 1

59655 Villeneuve DAscq Cedex

France

e-mail : lescure@math.univ-lille1.fr 